\title{
Mirizzi Syndrome with Type F Bile Duct Anomaly- A Rare Case Report
}

\author{
Karthikeyan Mahalingam ${ }^{1 *}$, Soundararajan L $^{1}$, Srinivasan \\ Muthukrishnan¹, Prabhakaran R², Srinivasan UP², Naganathbabu $\mathrm{OL}^{3}$ \\ and Rajajeyakumar $\mathbf{M}^{4}$ \\ ${ }^{1}$ Senior resident, Institute of Surgical Gastroenterology, Rajiv Gandhi Government \\ General Hospital, Madras Medical College, Chennai, India
}

Case Report

Volume 2 Issue 4

Received Date: July 05, 2018

Published Date: July 31, 2018

DOI: $10.23880 /$ mjccs- 16000165

${ }^{2}$ Assistant professor, Institute of Surgical Gastroenterology, Rajiv Gandhi Government General Hospital, Madras Medical College, Chennai, India

${ }^{3}$ Director and Professor, Institute of Surgical Gastroenterology, Rajiv Gandhi Government General Hospital, Madras Medical College, Chennai, India

${ }^{4}$ Assistant Professor, Physiology, Trichy SRM Medical College Hospital \& Research Centre, (Affiliated by Dr. MGR Medical University, Chennai), Trichy, Tamilnadu, India - 621105

*Corresponding author: Karthikeyan Mahalingam, Senior resident Institute of Surgical Gastroenterology, Rajiv Gandhi Government General Hospital, Madras Medical College, Chennai, India, Tel: +919894224021; Email: truculentsmktvmc@gmail.com

\section{Abstract}

The Mirizzi's syndrome is an uncommon condition characterized by obstructive jaundice secondary to a gallstone impacted in the infundibulum of gallbladder obstructing the common bile duct. Pre-operative diagnosis is very difficult since clinical findings, laboratory data and radiological features are not specific. We are presenting a 36 year old female presented with obstructive jaundice, cholangitis and acute kidney injury. She was found to have choledocholithiasis with type F biliary anomaly and was managed conservatively with antibiotics and ERCP with biliary stenting. The patient was stabilized and subsequently taken up for surgery. Intra-operatively a type F biliary anomaly with Mirizzi syndrome was made out. Hence cholecystectomy with hepaticojejunostomy including the aberrant duct was done. The case is presented for the interesting and rare association of bile duct anomaly with Mirizzi's syndrome.

Keywords: Mirizzi Syndrome; Bile Duct Anomaly; Infundibulum; Choledocholithiasis; Hepaticojejunostomy 


\section{Case Report}

A 36 year old woman was admitted with clinical features of obstructive jaundice and cholangitis with acute kidney injury. MRCP showed shrunken gall bladder with multiple calculi and dilated CBD with calculi. An ERCP showed pus draining from ampulla and biliary sphincterotomy with stenting done in view of cholangitis which failed to respond by conservative measures. She was managed with antibiotics and conservatively managed till cholangitis and AKI resolved. She improved clinically and discharged with advice to return for definitive surgery 6 weeks later.

However, she came back2 months later with pain right hypochondrium and intermittent fever for 2 weeks. On examination, the patient was febrile, non icteric with tenderness in the right hypochondrium. Laboratory investigations revealed elevated total count of 18900 cells/cu.mm. MRCP revealed features of chronic calculus cholecystitis with multiple calculi in CBD and CHD with biliary stent in situ. ERCP and balloon trawling done and 8 stones were removed. CBD stent was exchanged.

Patient was readmitted after two weeks for definitive surgery. Her total counts, liver and renal function tests were within normal limits. An USG abdomen showed multiple GB stones, multiple stones in CBD which was dilated $(12 \mathrm{~mm})$. Redo MRCP showed pneumobilia, evidence of linear stent in LHD, CHD and CBD; multiple calculi noted in GB, cystic duct, CHD, proximal CBD and Right posterior sectoral duct which was found to be joining the cystic duct (type F), diameter of RHD, LHD, CHD were $7 \mathrm{~mm}, 8 \mathrm{~mm}, 14 \mathrm{~mm}$ respectively with a CBD diameter of $19 \mathrm{~mm}$ proximally and $2.6 \mathrm{~mm}$ distally with narrowing.

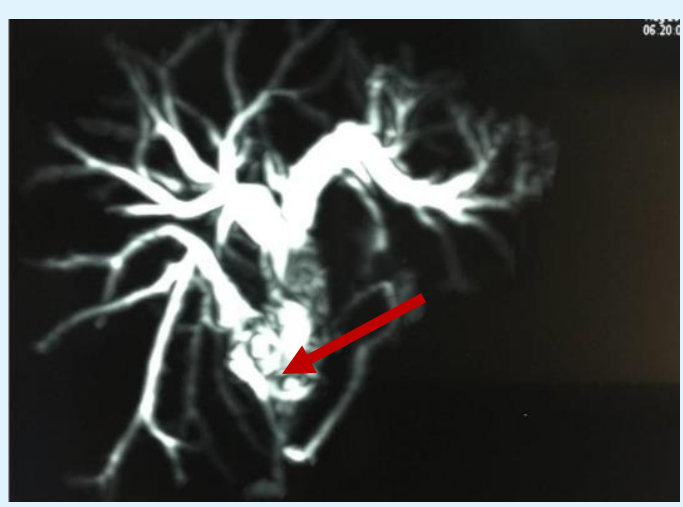

Figure 1: MRCP Showing Right Posterior Sectoral Duct Joining Cystic Duct (Type F Anomaly) With Mirizzi Syndrome Csendes Type 3.

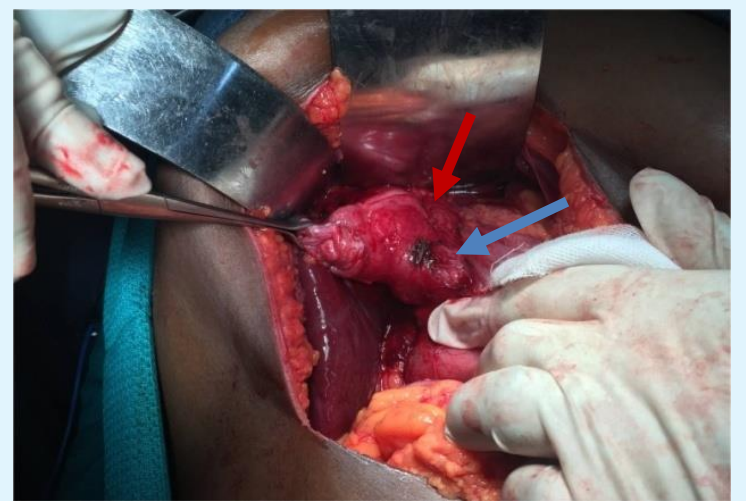

Figure 2: Intra Operative Picture Demonstrating Type 3 Mirizzi Syndrome (Blue Arrow) With Type F Bile Duct Anomaly Right Aberrant Duct Joining Cystic Duct (Red Arrow).

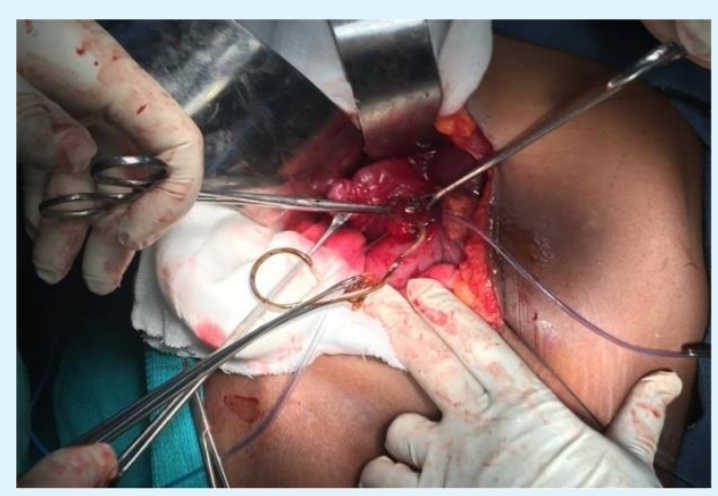

Figure 3: Common Bile Duct Stent Being Removed.

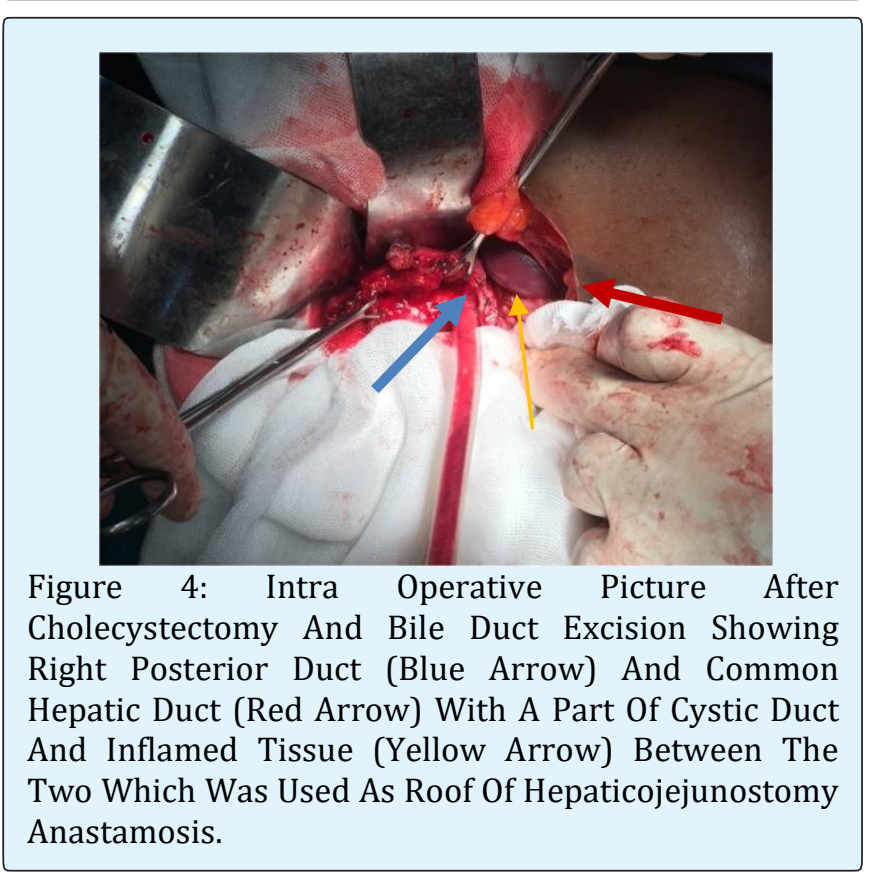



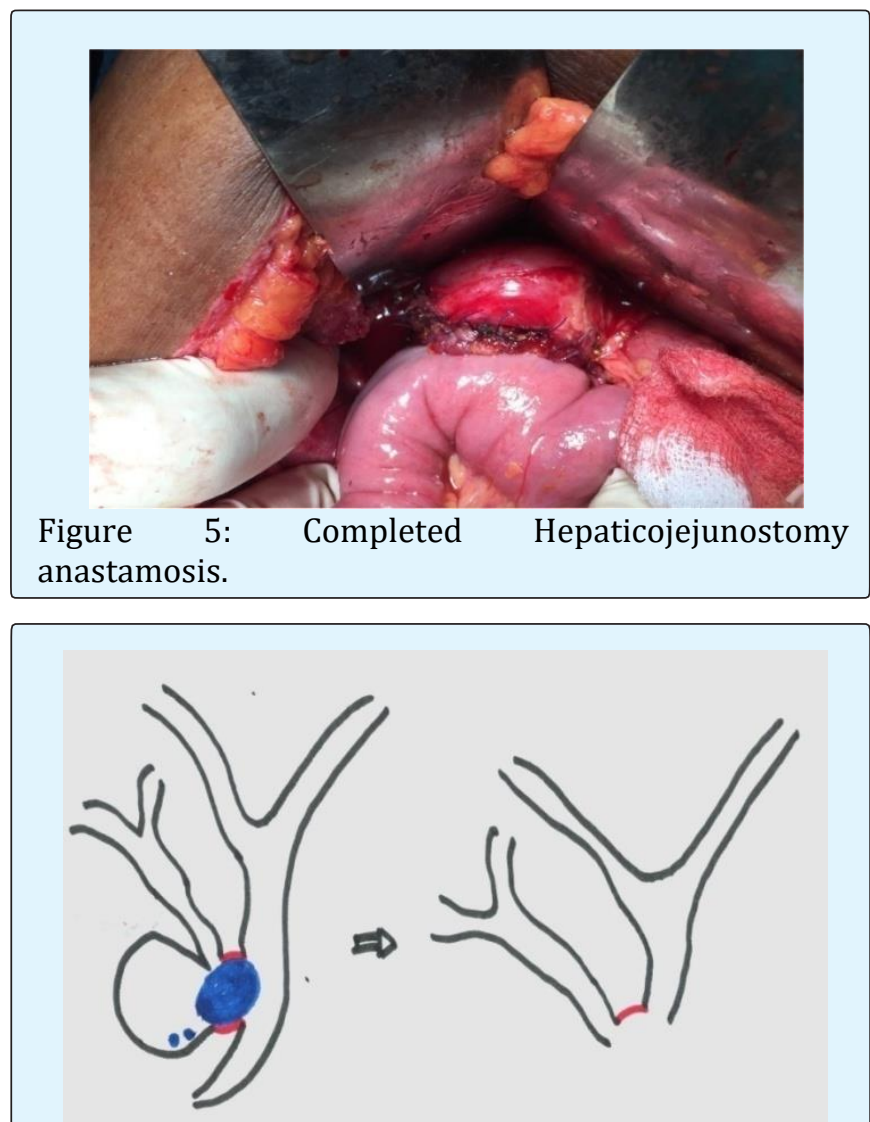

Figure 6: Simple pictorial representation of the Type F anomaly. Part of the cystic duct which is used as roof of hepaticojejunostomy is shown in red, stone yellow.
She was planned for an open cholecystectomy and CBD exploration. Intraoperatively, she was found to have a contracted gall bladder with multiple stones and stones in the CHD, CBD and aberrant duct (Type F bile duct anomaly -Rt posterior sectoral duct joining Cystic duct). CBD exploration done, stone and stent retrieval was done. Cholecystectomy was done. Aberrant Right posterior sectoral duct and CHD identified and stones were removed. Roux en Y hepaticojejunostomy was done including CHD and right posterior sectoral duct in single anastamosis (Figures 4 \& 5). She had an uneventful recovery in the postoperative period.

\section{Discussion}

Mirizzi syndrome is a rare complication of long standing cholelithiasis which occurs in 0.5 to $4 \%$ people with gall stone disease [1]. It is more common in females between 21 to 90 years old [1]. The first description about such condition was made by Pablo Luis Mirizzi in 1940 but Mirizzi believed that the condition was more physiological than mechanical, for which he thought that the stone causes inflammatory reaction that stimulates the sphincter in the common hepatic duct resulting in obstruction [2-6]. But later studies proved that there are no sphincters in common hepatic duct and the syndrome is due to mechanical obstruction of biliary tract due to stones impacted in Hartmann's, infundibulum or cystic duct causing inflammation and compression of biliary tract $[3,6]$.

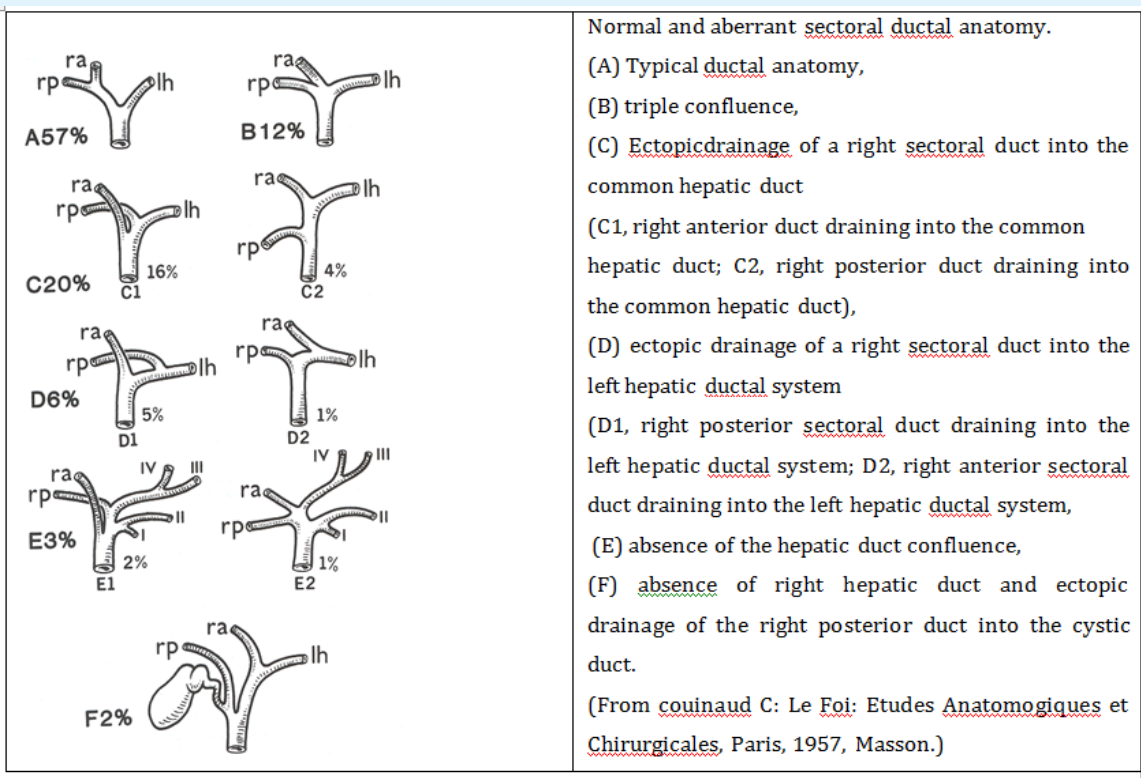

Figure 7: Normal and aberrant sectoral ductal anatomy. 


\section{Medical Journal of Clinical Trials \& Case Studies}

The pathophysiology of Mirizzi syndrome includes the external compression of the common duct and the continuing inflammatory process leads to cholecystobiliary and cholecystoenteric fistulas [7]. The anatomical factors include stones in the Hartmann's pouch with long cystic duct, cystic duct parallel to the common duct, low insertion of cystic duct are more prone to develop Mirizzi syndrome [7]. Symptoms of Mirizzi syndrome include abdominal pain, fever with chills and jaundice as in biliary obstruction. Stones migrating into intestinal tract either distally resulting in intestinal obstruction called as biliary ileus or may also rarely produce proximal duodenal obstruction causing features of gastric outlet obstruction a clinical condition described in literature as Bouveret syndrome [8,9] (Figure 7).

Pre-operative diagnosis is important for better management of mirizzi syndrome and also to prevent injuries during surgery. Since the syndrome is not associated with a defined set of clinical features, imaging is needed for diagnosis and management. Previously ERCP was considered essential for diagnosis of mirizzi syndrome but now MRCP is considered better non invasive technique for the diagnosis [10]. MRCP will be a better imaging modality to diagnose Mirrizi syndrome based on the following criteria: Dilated biliary tree above the level of GB neck, collapsed below that level with impacted stone in gall bladder neck or infundibulum, sometimes it present like a inverted meniscus sign $[11,12]$. Pre operative diagnosis and classification of type is important as the management and surgical procedure depends on the type of mirizzi syndrome. While simple cholecystectomy is enough for Csendes type 1 mirizzi syndrome which does not erode into bile duct other types will require some form bile duct repair with Csendes type 4 mostly requiring bilio-enteric anastomosis [13].

Mirizzi syndrome per se is difficult to diagnose and treat: the presence of bile duct anomaly further complicates the management. Mirizzi syndrome associated with bile duct anomaly is very rarely reported in the literature. Our patient is type 3 Mirizzi with type F bile duct anomaly in which the aberrant right hepatic duct joins the cystic duct. Because this condition was diagnosed pre operatively, a prompt repair could be done there by preventing biliary fistula due to missed aberrant right hepatic duct. Due to inflammation the aberrant duct joining cystic duct was found to be adherent to common hepatic duct. So cystic duct was ligated lateral to the junction of the aberrant duct with cystic duct, allowing for wide hepaticojejunostomy (Figure 4). Thus separate anastamosis of aberrant duct with intestine is avoided.

\section{Conclusion}

Although Mirizzi syndrome is rare, uncommon association of mirizzi syndrome with bile duct anomaly should also be taken in to consideration while operating upon such cases and prompt caution and better understanding of anatomy by preoperative evaluation will give better results.

\section{References}

1. Lacerda PS, Ruiz MR, Melo A, Guimarães LS, SilvaJunior RA, et al. (2014) Mirizzi Syndrome: A Surgical Challenge, Abcd Arq Bras Cir Dig 27(3): 222-231.

2. Martnez MA, Granero LE, Mirizzi PL (2009) Acta Gastroenterol Latinoam 39(3): 177-178.

3. Leopardi LN, Maddern GJ (2007) Pablo Luis Mirizzi: the man behind the syndrome. ANZ J Surg 77(12): 1062-1064.

4. Mirizzi PL (1940) Physiologic sphincter of the hepatic bile duct. Arch Surg 41: 1325-1333.

5. Mirizzi PL (1948) Sndrome del conducto heptico. J Int Chir 8: 731-777.

6. Lai EC, Lau WY (2006) Mirizzi syndrome: history, present and future development. ANZ J Surg 76(4): 251-257.

7. Unadkat $P$, Varma R, Gaikwad $K$, Halgaonkar P, Vaja C (2016) Mirizzi Syndrome: A Case Report. IJSS Case Reports \& Review 2: 42-4.

8. Torres OJM, Melo LAL, Rodrigues CEC (2002) Mirizzi's Syndrome. Rev do Hosp Univ UFMA 3(1): 41-43.

9. Iniguez A, Butte JM, Zuniga JM, Crovari F, Llanos O (2008) Sindrome de Bouveret. Resolucion endoscopicay quirurgica de cuatro casos clinicos. Revista Medica de Chile 136(2): 163-168.

10. Pyo NK, Outwater EK, Mitchell DG (1999) Mirizzi syndrome: evaluation by MR imaging. Am J Gastroenterol 94(9): 2546-2550.

11. Becker CD, Hassler H, Terrier F (1984) Preoperative diagnosis of the Mirizzi syndrome: limitations of sonography and computed tomography. AJR Am J Roentgenol 143(3): 591-596. 


\section{Medical Journal of Clinical Trials \& Case Studies}

12. Mithani R, Schwesinger WH, Bingener J, Sirinek KR, Gross GW (2008) The Mirizzi syndrome: multidisciplinary management promotes optimal outcomes. J Gastrointest Surg 12(6): 1022-1028.

13. Waisberg J, Corona A, De Abreu IW, Farah JF, Lupinacci RA, et al. (2005) Benign obstruction of the common hepatic duct (Mirizzi syndrome): Diagnosis and operative management. Arq Gastroenterology 42(1): 13-18. 